\title{
Reprogrammable logic-memory device of a mechanical resonator
}

\author{
AUTHOR(S):
}

Yao, Atsushi; Hikihara, Takashi

\section{CITATION:}

Yao, Atsushi ...[et al]. Reprogrammable logic-memory device of a mechanical resonator. International Journal of Non-Linear Mechanics 2017, 94: 406-416

\section{ISSUE DATE:}

2017-09

URL:

http://hdl.handle.net/2433/219611

\section{RIGHT:}

(C2016. This manuscript version (Preprint) is made available under the CC-BY-NC-ND 4.0 license http://creativecommons.org/licenses/by-nc-nd/4.0/; This is not the published version. Please cite only the published version.; この論文は出版社版でありません。引用 の際には出版社版をご確認ご利用ください。 


\title{
Reprogrammable logic-memory device of a mechanical resonator
}

\author{
Atsushi Yao ${ }^{\mathrm{a}}$, Takashi Hikihara ${ }^{\mathrm{b}}$ \\ ${ }^{a}$ Department of Electronic Science and Engineering, Kyoto University, Katsura, Nishikyo, \\ Kyoto, 615-8510 Japan \\ ${ }^{b}$ Department of Electrical Engineering, Kyoto University, Katsura, Nishikyo, Kyoto, \\ 615-8510 Japan
}

\begin{abstract}
From the viewpoint of application of nonlinear dynamics, we report multifunctional operation in a single microelectromechanical system (MEMS) resonator. This paper addresses a reprogrammable logic-memory device that uses a nonlinear MEMS resonator with multi-states. In order to develop the reprogrammable logic-memory device, we discuss the nonlinear dynamics of the MEMS resonator with and without control input as logic and memory operations. Through the experiments and numerical simulations, we realize the reprogrammable logic function that consists of OR/AND gate by adjusting the excitation amplitude and the memory function by storing logic information in the single nonlinear MEMS resonator.

Keywords: micro-electro-mechanical resonator, nonlinear dynamics, logic-memory device, logic operation, memory operation, nonlinear vibrations
\end{abstract}

\section{Introduction}

A mechanical computer consists of mechanical components such as gears, beams and so on. The history of mechanical computation began when the mechanical calculator was invented by Wilhelm Schickard in the 17th century. In 1644, Blaise Pascal designed and built a small and simple mechanical calculator

Email addresses: yao@piezo.kuee.kyoto-u.ac.jp (Atsushi Yao), hikihara.takashi.2n@kyoto-u.ac.jp (Takashi Hikihara) 
(called Pascaline) that consisted of the fundamental operations of addition and subtraction [1-3]. In the 19th century, Charles Babbage tried to build the analytical engine that had the concept of a programmable computer, the automatic storage and retrieval of information in coded form, the automatic execution of a sequence of operations, and so on $[1,2,4-6]$. Thereafter, many mechanical calculators continued to be utilized until the 1960's. However, mechanical calculators were replaced by electronic calculators in the early 1970's [3, 7]. Do the histories of mechanical computation finish?

Microelectromechanical systems or nanoelectromechanical systems (MEMS or NEMS) devices have micro-scale or nano-scale dimensions and mainly contain both electrical and mechanical components. MEMS and NEMS devices have been extensively studied and used as novel functional devices such as accelerometers [8], ink jet nozzle printing arrays [9], radio-frequency (RF) MEMS [10-12], and so on $[13,14]$. In many MEMS devices, we focus on MEMS resonators. In the 1960's, Harvey C. Nathanson et al. produced the first MEMS resonator that was called resonant gate transistor $[15,16]$. In recent years, MEMS and NEMS resonators have been used as frequency references, sensor elements, and filters due to high quality factor (Q factor) [14].

Recently, mechanical computation based on MEMS or NEMS resonators has attracted great attention for its potential applications [17-38]. In particular, we have demonstrated a "logic-memory operation" that offers a combination of OR gate and memory operations in a single nonlinear MEMS resonator [35]. Previous studies have reported that the mechanical resonator can be used as reprogrammable logic devices $[21,24,26,30,38]$. The next phase is to develop a "reprogrammable logic-memory device" in the single nonlinear MEMS resonator.

Most modern computers use a binary representation that has two states "1" and "0". In 1987, M. V. Andres and colleagues showed that a MEMS resonator exhibited nonlinear responses such as bistable and hysteretic characteristics [39]. The nonlinear dynamical responses are commonly observed in a MEMS or NEMS resonator $[13,14,40,41]$. The nonlinear dynamics of the 
resonator is well known to be described by the Duffing equation [17, 23, 28, 4244]. Such a nonlinear resonator has hysteretic characteristics with respect to the excitation frequency $[13,14]$ or excitation force $[21,31]$. In the hysteretic region, the MEMS resonator exhibits two coexisting stable states that correspond to large and small amplitude vibrations [17, 21, 23, 28, 31, 42-44]. Thus, a nonlinear MEMS resonator can work as a 1-bit mechanical memory or logic device indicating "1" and "0" at large and small vibrations.

From the viewpoint of application of nonlinear dynamics in the MEMS resonator, this paper numerically and experimentally demonstrates the reprogrammable logic gate and the memory operations as s multifunctional device. The nonlinear dynamics of the MEMS resonator with and without control input is examined as logic and memory operations. Furthermore, in order to realize the reprogrammable logic-memory device, the nonlinear dynamics is examined at the change of excitation amplitude in the MEMS resonator. A preliminary version of this work was published in [36, 37]. The present paper contains experimental results for the reprogrammable logic gate that was not included in the preliminary work. Here, we show our integrated paper that includes the reprogrammable device based on our measurement, control, and logic-memory system.

The rest of this paper is organized as follows. Section 2 and 3 present a fabricated MEMS resonators and its dynamical model, respectively. Section 4 explains the control system to perform the logic and memory operations in the nonlinear MEMS resonator. In Section 5 and 6, the reprogrammable logicmemory operation is numerically and experimentally achieved. In Section 7, the conclusions of this paper are summarized.

\section{MEMS resonator and its measurement system}

This section explains a fabricated MEMS resonator and a displacement measurement of the comb-drive resonator by using the differential measurement. 


\subsection{Schematic of comb-drive resonator}

Figure 1 shows a fabricated comb-drive MEMS resonator [43, 45, 46]. Fig. 1(a) shows a top view of the resonator. A cross section at the dotted lines of Fig. 1(a) is shown in Fig. 1(b). The MEMS resonator is fabricated using SOIMUMPs, which is a kind of silicon on insulator (SOI) technology and is offered by Memscap, Inc [47]. In this process, the MEMS device consists of a $25 \mu \mathrm{m}$ thick silicon layer as the structure layer, a $2 \mu \mathrm{m}$ thick oxide layer as the insulating layer, and a $400 \mu \mathrm{m}$ thick silicon layer as the substrate layer. The MEMS resonator has a perforated mass whose width, length, and thickness are 175,575 , and $25 \mu \mathrm{m}$, respectively and that are connected to four beams. The folded beams are designed to work as springs and are connected to anchors. The MEMS resonator has two (left and right) comb capacitors that are connected to the each side electrode, respectively. When the anchor and the electrode are connected to ground and to ac voltage source with a dc bias voltage, the mass vibrates primarily in the lateral direction ( $X$-direction) with weak link to the longitudinal and vertical directions.

\subsection{Measurement system}

By using the differential measurement as shown in Fig. 2, we measure the vibrations of the resonator. That is, the voltage of right (left) electrode is excited by $V_{1}=V_{\text {dce }}+v_{\mathrm{ac}} \sin 2 \pi f_{\mathrm{e}} t\left(V_{2}=V_{\mathrm{dce}}-v_{\mathrm{ac}} \sin 2 \pi f_{\mathrm{e}} t\right)$, where $v_{\mathrm{ac}}$ denotes the ac excitation voltage, $V_{\text {dce }}$ is the experimental dc bias voltage, and $f_{\mathrm{e}}$ is the experimental excitation frequency. In the differential measurement, when the displacement $x(t)$ is assumed as $A_{0} \sin \left(2 \pi f_{\mathrm{e}} t+\phi\right)$, where $A_{0}$ denotes the displacement amplitude and $\phi$ is the phase, the excitation force $F_{\text {alle }}$ and current $i$ are obtained as follows [27]:

$$
\begin{aligned}
F_{\text {alle }} & =4 \varepsilon N \frac{h}{d} V_{\mathrm{dce}} v_{\mathrm{ac}} \sin 2 \pi f_{\mathrm{e}} t, \\
i & =8 \pi \varepsilon N \frac{h}{d} f_{\mathrm{e}} v_{\mathrm{ac}} A_{0} \sin \left(4 \pi f_{\mathrm{e}} t+\phi\right),
\end{aligned}
$$

where $d(=3 \mu \mathrm{m})$ denotes the gap between the fingers, $l(=100 \mu \mathrm{m})$ is the initial overlap between the fingers, $\varepsilon\left(=8.85 \times 10^{-12} \mathrm{~F} / \mathrm{m}\right)$ is the permittivity, 
$N(=39)$ is the comb number, and $h(=25 \mu \mathrm{m})$ is the finger height. Hereafter, the ac excitation voltage is set to $0.5 \mathrm{~V}$. Note that the excitation force $F_{\text {alle }}$ is proportional to the dc bias voltage $V_{\text {dce }}$ in our experiments. Tab. 1 shows the device parameters. As a result, in the MEMS resonator, the comb drive serves as a forcing actuator, but which simultaneously serves as a displacement sensor.

Here, we consider the experimental system to measure the sum of the current $i$ through the right and left capacitors. Fig. 2 shows schematic diagram of measurement system to detect the current $i$ that is converted to the output voltage $V_{\text {out }}$ by two operational amplifiers (Burr-Brown; OPA627AP). The first (second) stage operational amplifier works as the current-to-voltage converter (inverting amplifier). The output voltage $V_{\text {out }}$ is obtained as

$$
V_{\text {out }}=R \frac{R_{2}}{R_{1}} i=8 \pi f_{\mathrm{e}} R \frac{R_{2}}{R_{1}} \varepsilon N \frac{h}{d} v_{\mathrm{ac}} A_{0} \sin \left(4 \pi f_{\mathrm{e}} t+\phi\right),
$$

where $R \quad(=1 \mathrm{M} \Omega), R_{1} \quad(=1 \mathrm{k} \Omega)$, and $R_{2} \quad(=100 \mathrm{k} \Omega)$ denote three resistors. It can be confirmed that $V_{\text {out }}$ depends on the amplitude $A_{0}$ and the phase $\phi$ of the displacement.

In our experiments, the voltages $\left(V_{1}\right.$ and $\left.V_{2}\right)$ of right and left electrodes are given by function generator (Tektronix; AFG3022). The output voltage is measured by an oscilloscope (Tektronix; DPO4104). The supply voltage of two operational amplifiers is set at $\pm 15 \mathrm{~V}$ and supplied by stabilized power supply (TEXIO; PW36-1.5AD). The mechanical vibrations are verified by using the

Table 1: Device parameters in experiments.

\begin{tabular}{cccc}
\hline Parameter & & Value & Unit \\
\hline$N$ & comb number & 39 & \\
$l$ & initial overlap between the fingers & 100 & $\mu \mathrm{m}$ \\
$h$ & height of the finger & 25 & $\mu \mathrm{m}$ \\
$d$ & gap between the fingers & 3 & $\mu \mathrm{m}$ \\
$\varepsilon$ & permittivity & $8.85418782 \times 10^{-12}$ & $\mathrm{~F} / \mathrm{m}$ \\
\hline
\end{tabular}


motion analysis microscope (KEYENCE; VW-6000).

The MEMS resonator is set in the vacuum chamber to reduce air resistance.

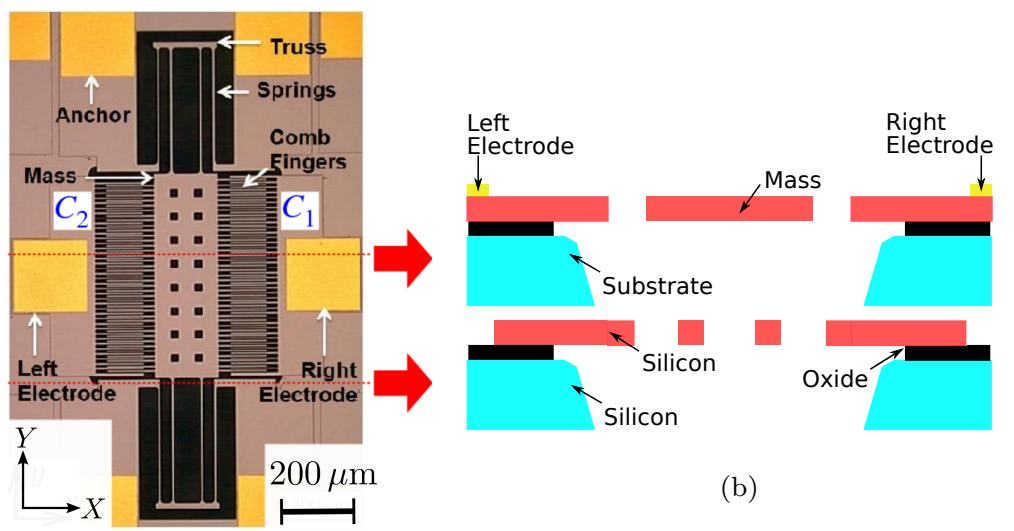

(a)

Figure 1: Schematic diagram of fabricated MEMS resonator: (a) Top view. (b) Cross section. The substrate and oxide layers underneath a movable structure are removed.

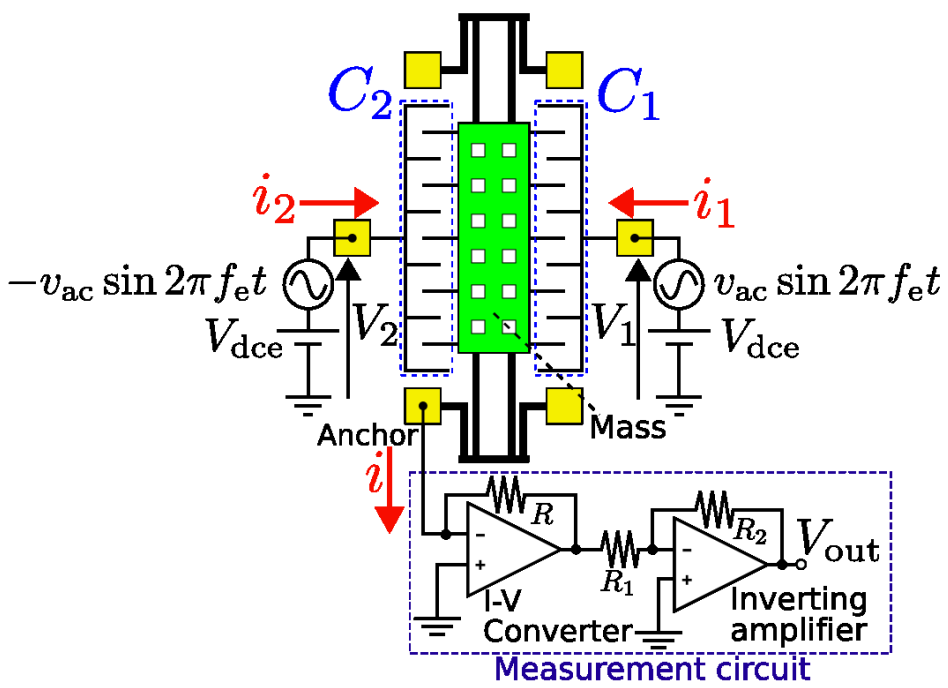

Figure 2: Schematic diagram of differential measurement. In the measurement system, the output voltage $V_{\text {out }}$ depends on the amplitude and phase of the displacement in the nonlinear MEMS resonator. 
The pressure is set at around $5 \mathrm{~Pa}$ at room temperature. In addition, in order to reduce an electrical noise due to a crosstalk, two operational amplifiers are also set in the vacuum chamber. The substrate and the vacuum chamber are grounded to reduce noise and parasitic capacitances.

Figure 3(a) shows the experimentally obtained frequency response curves of the displacement at $V_{\text {dce }}=150 \mathrm{mV}$. In Fig. 3(a), red and aqua lines show the response at upsweep and downsweep of frequency, respectively. We obtain quasi-static responses to continuous excitation in the hysteresis region at each excitation frequency. When the excitation frequency is set at $8.6600 \mathrm{kHz}$, the steady state is measured. Then, the excitation frequency is changed to higher value and we again obtain the steady state. Here, the response at the upsweep of frequency (red line) from $8.6600 \mathrm{kHz}$ to $8.6680 \mathrm{kHz}$ is measured. The downsweep test is done in the same manner as the upsweep test. We have confirmed that the MEMS resonator has hysteresis characteristics to frequency and two stable states coexist in the hysteresis region. Two stable states coexist at $8.6620 \mathrm{kHz}$ $<f_{\mathrm{e}}<8.6652 \mathrm{kHz}$. In the proposed device, when the excitation amplitude and/or the quality factor increases, the bandwidth of the hysteresis region also increases [46]. In the following experiments, the excitation frequency is fixed at $8.6622 \mathrm{kHz}$. Note that the difference between the large and small vibrations is small at $8.6622 \mathrm{kHz}$ in the hysteresis region and then feedback values as described in the following sections become small. In the next section, the excitation frequency is numerically discussed.

Figure 3(b) shows the experimentally obtained hysteretic behavior with respect to the dc bias voltage at $8.6622 \mathrm{kHz}$. The hysteresis region exists at $30 \mathrm{mV}$ $<V_{\text {dce }}<150 \mathrm{mV}$. In our study, these stable regions, which have large and small amplitude vibrations, define the two states of the single-output logic-memory device in a single MEMS resonator. As a result, we realize the read operation by using the differential measurement. In our experiments, for memory and logic output, the thin (aqua) and dark (red) lines are regarded as a logical "1" and logical "0", respectively. 


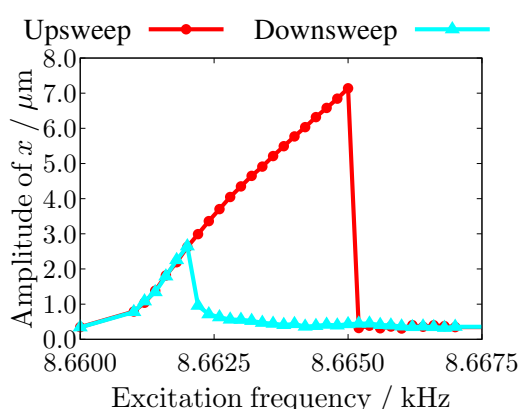

(a)

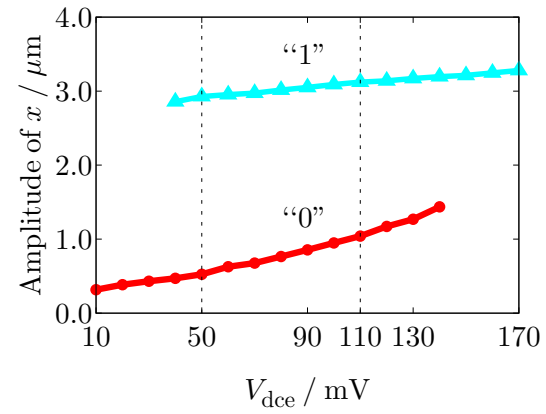

(b)

Figure 3: Hysteretic characteristics (Experimental results). In (a) ((b)), the red and aqua lines correspond to the responses at the upsweep and the downsweep of frequency (excitation amplitude), respectively. In the hysteretic regime, the resonator can exist in two distinct amplitude states: (a) Amplitude-frequency response curves of resonator at $v_{\mathrm{ac}}=0.5 \mathrm{~V}$ and $V_{\text {dce }}=150 \mathrm{mV}$. (b) Hysteretic characteristics as a function of dc bias voltage at $8.6622 \mathrm{kHz}$.

\section{Dynamical model and two stable states}

This section addresses the dynamical model of the nonlinear MEMS resonator and its two stable vibrations. In addition, the convergence conditions of two stable states are examined.

\subsection{Dynamical model and its steady state}

The nonlinear dynamics of the MEMS resonator is modeled by the Duffingtype equation as follows:

$$
\begin{aligned}
& \frac{\mathrm{d}^{2} x}{\mathrm{~d} t^{2}}+\frac{2 \pi f_{0}}{Q} \frac{\mathrm{d} x}{\mathrm{~d} t}+\left(2 \pi f_{0}\right)^{2} x+\alpha_{3} x^{3}=\frac{F_{\text {alln }}}{m_{\mathrm{e}}} \sin 2 \pi f_{\mathrm{n}} t, \\
& \frac{\mathrm{d}^{2} x}{\mathrm{~d} t^{2}}+\frac{2 \pi f_{0}}{Q} \frac{\mathrm{d} x}{\mathrm{~d} t}+\left(2 \pi f_{0}\right)^{2} x+\alpha_{3} x^{3}=4.0 \mathrm{~m}\left(\mathrm{Vs}^{2}\right)^{-1} \times V_{\mathrm{dcn}} \sin 2 \pi f_{\mathrm{n}} t,
\end{aligned}
$$

where $x$ denotes the displacement, $f_{0}(=8.6644 \mathrm{kHz})$ is the resonance frequency, $Q(=25,000)$ is the quality factor, $\alpha_{3}\left(=7.06 \times 10^{16}(\mathrm{sm})^{-2}\right)$ is the coefficient of cubic correction to the linear restoring force, $f_{\mathrm{n}}$ is the numerical excitation frequency, $V_{\mathrm{dcn}}$ is the dc bias voltage for numerical simulations, $m_{\mathrm{e}}(=1.72 \times$ $\left.10^{-9} \mathrm{~kg}\right)$ is the effective mass, and $F_{\text {alln }}\left(=6.906 \times 10^{-9} \mathrm{NV}^{-1} \times V_{\mathrm{dcn}}\right)$ is the 
excitation amplitude for the numerical simulations. The parameter settings are obtained from Refs. [35, 37], which deal with the same device as depicted in Fig. 1.

Figure 4(a) shows numerical amplitude-frequency response curves generated from Eq. (4) at $V_{\mathrm{dcn}}=150 \mathrm{mV}$. The model of the MEMS resonator exhibits a hysteretic response. The solid (red and aqua) lines show two stable solutions and the dashed (green) line shows an unstable solution. In our numerical simulations, the unstable solution is obtained by a shooting method [48]. At any given frequency in the hysteretic region, the MEMS resonator has two coexisting stable states. Two states are obviously distinguished.

Figure 4(b) shows the numerically determined hysteretic behavior as a function of the dc bias voltage $V_{\mathrm{dcn}}$ related to excitation force at $f_{\mathrm{n}}=8.6654 \mathrm{kHz}$. The hysteresis region appears at $105 \mathrm{mV}<V_{\mathrm{dcn}}<245 \mathrm{mV}$ in Fig. 4(b). The nonlinear MEMS resonator has stable regions (solid line) that are completely separated by an unstable region (dashed line). These stable regions can be used as two states, corresponding to logical " 0 " and "1", for memory function. In the numerical simulations, a displacement amplitude greater (less) than $3.0 \mu \mathrm{m}$ is regarded as a logical "1" ("0") for memory and logic output. As a result, we numerically demonstrate the read operation of memory device that consists of the MEMS resonator. The numerical results in Figs. 4(a) and (b) are in agreement with experimental results in Figs. 3(a) and (b).

\subsection{Basins of attractions}

This section focuses on the multiplicity of alternative stable attracting solutions. The solutions depend on the initial conditions, which correspond to the displacement $x$ and the velocity $y$ (or the amplitude and the phase) [49]. A study of stroboscopic points of the $x y$ plane onto itself serves to determine the basins of attraction $[49,50]$ of the original continuous differential equation Eq. (4). Here, a stroboscopic map [51] is considered.

The nonlinear dynamics of the MEMS resonator is described by the differ- 


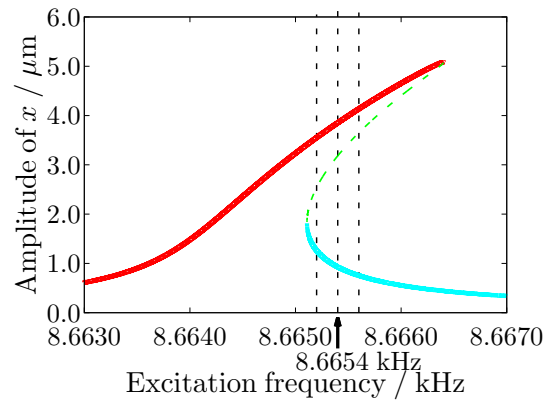

(a)

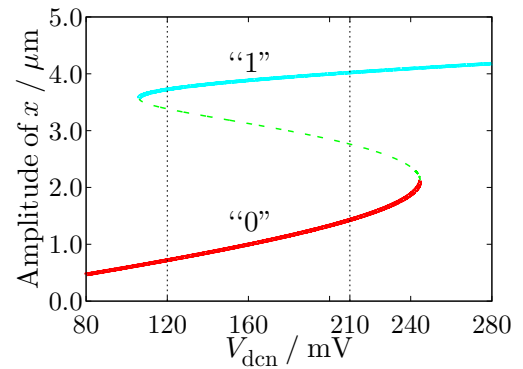

(b)

Figure 4: Corresponding hysteretic characteristics with Eq. (4) (Numerical results). The solid (red and aqua) lines show two stable solutions and the dashed (green) line shows an unstable solution: (a) Numerical amplitude-frequency response curves at $V_{\mathrm{dcn}}=150 \mathrm{mV}$. (b) Numerical hysteretic characteristics as a function of dc bias voltage $V_{\mathrm{dcn}}$ at $f_{\mathrm{n}}=8.6654 \mathrm{kHz}$.

ential equations with the velocity $y$ :

$$
\left\{\begin{array}{l}
\frac{\mathrm{d} x}{\mathrm{~d} t}=y \\
\frac{\mathrm{d} y}{\mathrm{~d} t}=-\frac{2 \pi f_{0}}{Q} \frac{\mathrm{d} x}{\mathrm{~d} t}-\left(2 \pi f_{0}\right)^{2} x-\alpha_{3} x^{3}+4.0 \mathrm{~m}\left(\mathrm{Vs}^{2}\right)^{-1} \times V_{\mathrm{dcn}} \sin 2 \pi f_{\mathrm{n}} t .
\end{array}\right.
$$

The initial conditions sampled by the period of the excitation frequency at $f_{\mathrm{n}}=8.6654 \mathrm{kHz}$ are considered as shown in Fig. 5. In the figure, the white and black regions show the basins of two stable solutions. The green and blue points correspond to the stroboscopic points sampled by the period of the excitation frequency. The green point shows the large amplitude solution and the blue point is the small amplitude solution. These two stable solutions possess their basins completely separated by stable manifolds of an unstable solution (aqua point). In white and black regions, every initial state converges toward individual solutions. In Fig. 6, the red (purple) points correspond to the locus of convergence to the large (small) amplitude solution in the $x y$ plane.

Figures 7(a) and (b) show the set of initial conditions sampled by the period of each excitation frequency that corresponds to $f_{\mathrm{n}}=8.6652 \mathrm{kHz}$ and $f_{\mathrm{n}}=$ $8.6656 \mathrm{kHz}$, respectively. In the figures, the green, blue, and aqua points denote the stroboscopic points that correspond to the large amplitude, small amplitude, 
and unstable solutions at each excitation frequency, respectively. The white to black ratio depends on the excitation frequency as shown in Figs. 5 and 7. In other words, the white region expands at the upsweep of frequency and vice versa.

As mentioned above, the basins of attraction are completely separated by stable manifolds of the unstable solution. In the nonlinear MEMS resonator, the basins of attraction tend to have a spiral form, which depends on both displacement and velocity. Here, two stable solutions have each basin of attraction around each solution. Therefore, the nonlinear MEMS resonator has the capability of staying at either of two stable solutions. In our numerical simulations, the numerical excitation frequency is set to $8.6654 \mathrm{kHz}$. In order to realize the logic operation in the nonlinear MEMS resonator, we need to pass across the stable manifolds by using the control input.

\section{Control system}

This section focuses on the control system to perform logic and memory operations in the nonlinear MEMS resonator with hysteresis at a single excitation frequency. Recently, we have demonstrated a logic-memory operation that offers a combination of OR gate and memory operations in a single nonlinear MEMS

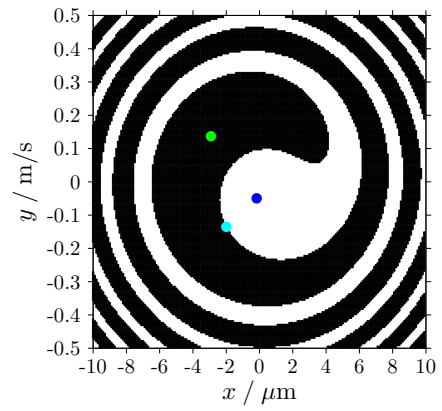

Figure 5: Basins of attraction at $f_{\mathrm{n}}=$ $8.6654 \mathrm{kHz}$.

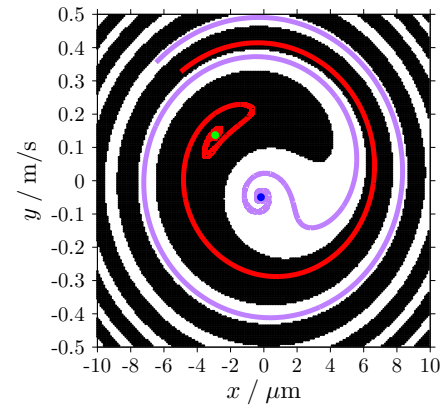

Figure 6: Basins of attraction at $f_{\mathrm{n}}=$ $8.6654 \mathrm{kHz}$. Red (purple) points have the initial state in black (white) region. 


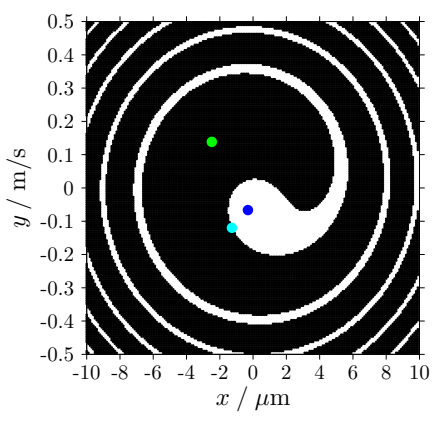

(a)

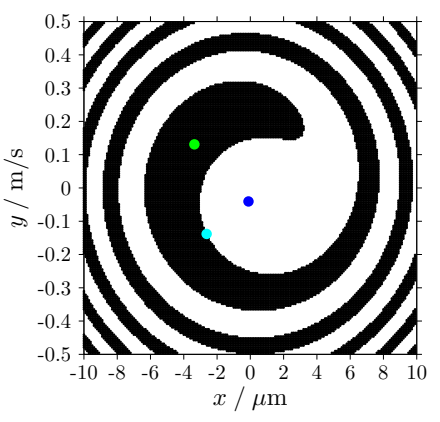

(b)

Figure 7: Basins of attraction: (a) $f_{\mathrm{n}}=8.6652 \mathrm{kHz}$. (b) $f_{\mathrm{n}}=8.6656 \mathrm{kHz}$.

resonator. To realize the logic-memory device, the output of the device does not depend on the previous values of the inputs and memory but the current values of the logic inputs [37]. To do this, we have applied the feedback control to the MEMS resonator [35].

\subsection{Experimental system}

Figure 8 shows the control system in the experiment that is implemented with a feedback and logic inputs. As studied above, in the differential measurement, the MEMS resonator can be equipped with a comb drive that serves as the forcing actuator and the displacement sensor. Here, to perform the control without any influence to measurement, the control input is applied as a slowly changing dc voltage $V_{\text {ave }}^{2}$ to the nonlinear MEMS resonator [27]. As shown in Fig. 8 , the output voltage $V_{\text {out }}$ is converted to the slowly changing dc voltage $V_{\text {ave }}^{2}$ by an analog multiplier (ANALOG DEVICES; AD633) and a low-pass filter of the operational amplifier (Burr-Brown; OPA627AP) whose time constant is set to about $0.47 \mathrm{~s}$. The logic inputs are represented by two dc voltages $\left(L_{\text {ine1 }}\right.$ and $L_{\text {ine2 }}$ ) for avoiding the influence to measurement. As a result, the feedback input $K_{\mathrm{e}} V_{\text {ave }}^{2}$ and two logic inputs $\left(L_{\mathrm{ine} 1}\right.$ and $\left.L_{\mathrm{ine} 2}\right)$ are added to the dc bias voltage $V_{\mathrm{dce}}$. 


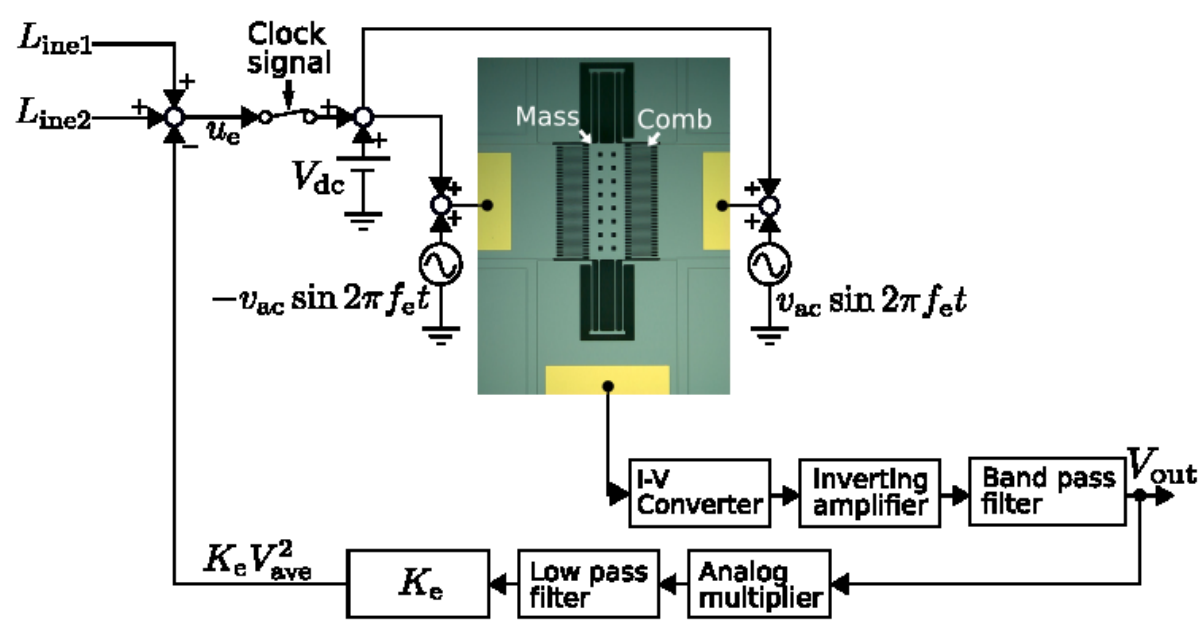

Figure 8: Schematic of control system that relates to logic inputs in experiments. When the experimental logic inputs $\left(L_{\mathrm{ine} 1}, L_{\mathrm{ine} 2}\right)$ are applied to the resonator, the excitation force is proportional to $V_{\text {dce }}+u_{\mathrm{e}}=V_{\mathrm{dce}}+L_{\mathrm{ine} 1}+L_{\mathrm{ine} 2}-K_{\mathrm{e}} V_{\text {ave }}^{2}$, where $u_{\mathrm{e}}$ is the control input and $K_{\mathrm{e}}\left(=11 \mathrm{~V}^{-1}\right)$ is the feedback gain in the experiments.

In the experiments, the control input $u_{\mathrm{e}}$ is described by

$$
u_{\mathrm{e}}=L_{\mathrm{ine} 1}+L_{\mathrm{ine} 2}-K_{\mathrm{e}} V_{\mathrm{ave}}^{2}
$$

The excitation force $F_{\text {alle }}$ under the control is described by

$$
F_{\text {alle }} \propto\left(V_{\text {dce }}+u_{\mathrm{e}}\right) \sin 2 \pi f_{\mathrm{e}} t
$$

\subsection{Numerical system}

Figure 9 shows the control system in our numerical simulations to perform the logic and memory operations in the nonlinear MEMS resonator. According to the experimental method, the numerical logic inputs $\left(L_{\text {inn1 }}\right.$ and $\left.L_{\text {inn2 }}\right)$ and the numerical feedback value $K_{\mathrm{n}} A_{\text {nave }}^{2}$ are added to the dc bias voltage $V_{\mathrm{dcn}}$ related to the excitation force. The nonlinear dynamics of the MEMS resonator with the corresponding numerical control input $u_{\mathrm{n}}$ is obtained by

$$
\frac{\mathrm{d}^{2} x}{\mathrm{~d} t^{2}}+\frac{2 \pi f_{0}}{Q} \frac{\mathrm{d} x}{\mathrm{~d} t}+\left(2 \pi f_{0}\right)^{2} x+\alpha_{3} x^{3}
$$




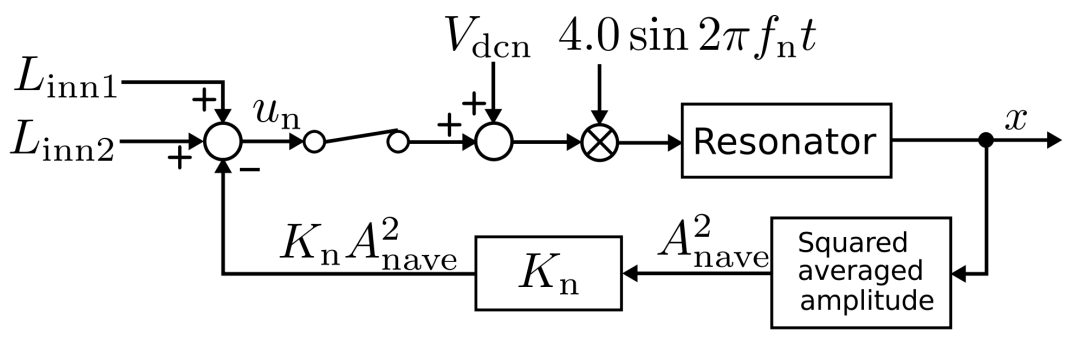

Figure 9: Control system in numerical simulations. $K_{\mathrm{n}}$ denotes the numerical feedback gain.

$$
\begin{array}{r}
=4.0 \mathrm{~m}\left(\mathrm{Vs}^{2}\right)^{-1} \times\left(V_{\mathrm{dcn}}+u_{\mathrm{n}}\right) \sin 2 \pi f_{\mathrm{n}} t, \\
u_{\mathrm{n}}=L_{\mathrm{inn} 1}+L_{\mathrm{inn} 2}-K_{\mathrm{n}} A_{\text {nave }}^{2}, \\
A_{\text {nave }}^{2}=\frac{A_{\mathrm{n} 1}^{2}+A_{\mathrm{n} 2}^{2}+\cdots+A_{\mathrm{n} m}^{2}+\cdots+A_{\mathrm{n} M}^{2}}{M},
\end{array}
$$

where $m$ denotes a natural number, $M$ is the average number, and $A_{\mathrm{n} m}$ is the displacement amplitude of the previous $m$ periods within $1 \leq m \leq M$ for the numerical simulations. In this case, $A_{\text {nave }}^{2}$ is the average of $A_{\mathrm{n} m}^{2} \cdot M$ is set to 22,000 and $K_{\mathrm{n}}$ is set to $1.42 \times 10^{10} \mathrm{Vm}^{-2}[35,37]$.

\section{Logic and memory operations}

\subsection{OR gate and memory operations}

Here, through the experiments and numerical simulations, we discuss both logic and memory operations in a MEMS resonator using the control system. In this section, we consider the dynamical model of the nonlinear MEMS resonator with and without control input [35]. Here, the dc bias voltage $V_{\text {dcn }}$ is set to 210 $\mathrm{mV}$.

Figures 10(a) and (b) show the amplitude modulation systematically varied in input signals $L_{\mathrm{inn} 1}$ and $L_{\mathrm{inn} 2}$ when the control input is applied to the MEMS resonator at $V_{\mathrm{dcn}}=210 \mathrm{mV}$. By applying the control input, a modulation of the resonator's amplitude is induced. In Fig. 10(a) ((b)), the initial state corresponds to logical " 1 " ("0") for memory output. In these figures, the light (dark) region corresponds to more than (less than) $3.0 \mu \mathrm{m}$ in displacement 


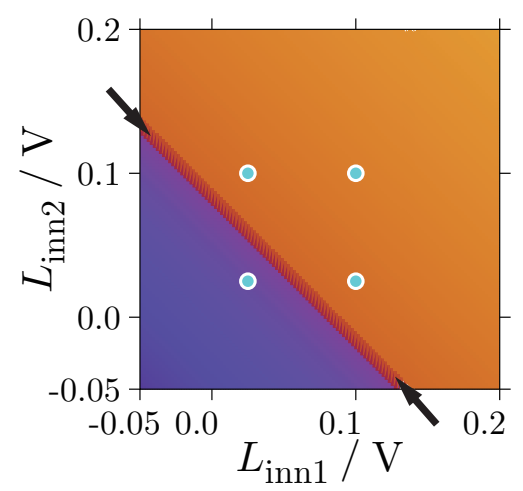

(a)

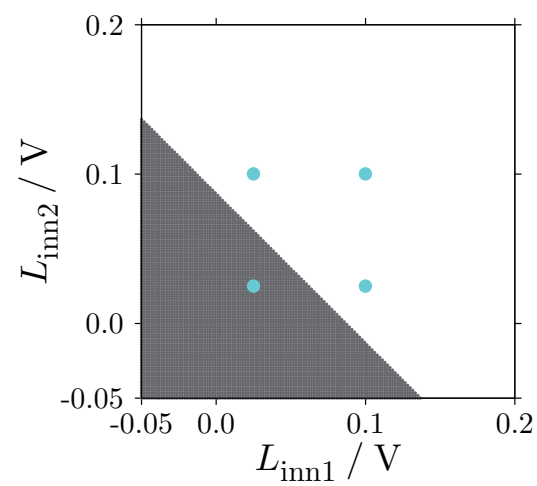

(c)

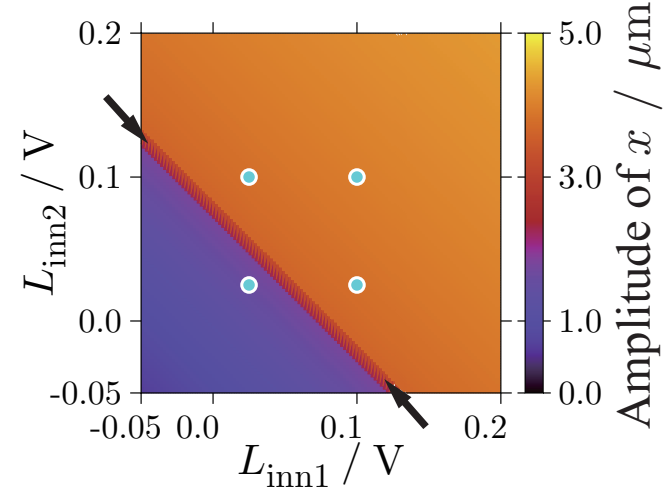

(b)

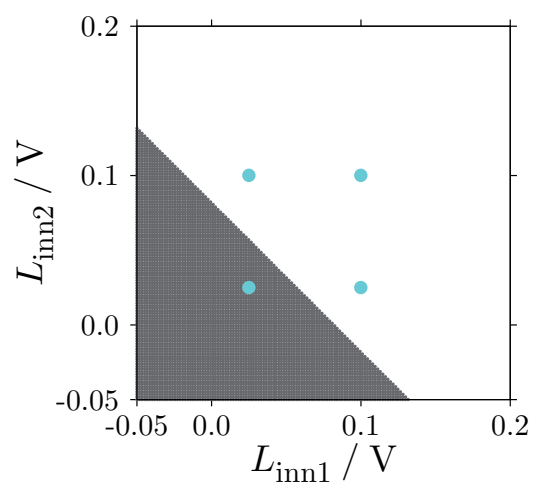

(d)

Figure 10: Displacement amplitude (logic output) with and without control input at $V_{\mathrm{dcn}}=$ $210 \mathrm{mV}$. (a) and (b) show the amplitude modulation systematically varied in input signals $L_{\mathrm{inn} 1}$ and $L_{\mathrm{inn} 2}$. (c) and (d) show the final state when the control input is off: (a) Initial state is the logical " 1 " for memory output. (b) Initial state corresponds to the logical "0". (c) Convergence conditions corresponding to Fig. 10(a). (d) Convergence conditions corresponding to Fig. 10(b).

amplitude, corresponding to a logical "1" (logical "0") output. In our numerical simulations, the logic input is regarded as logical 1 (logical 0) when the input signal $\left(L_{\mathrm{inn} 1}\right.$ or $\left.L_{\mathrm{inn} 2}\right)$ is set to $100 \mathrm{mV}(25 \mathrm{mV})$, as shown by the four (aqua) circles in Figs. 10(a) and (b). Therefore, the nonlinear MEMS resonator can be utilized as an OR gate at $V_{\mathrm{dcn}}=210 \mathrm{mV}$ as shown in Tab. 2 .

Next, the memory operation is considered in the nonlinear MEMS resonator. 
Figs. 10(c) and (d) show the calculated convergence conditions when the control inputs shown in Figs. 10(a) and (b) are off. As shown in Fig. 6, the convergence condition depends on basins of attraction. Two stable solutions have each basin of attraction around each solution under these conditions. Therefore, the initial state cannot switch to the other stable state and converges to the original stable state due to the basins of attraction. The white (black) region corresponds to convergence to the large (small) amplitude solution, corresponding to a logical "1" ("0") for memory output. The light (dark) region in Figs. 10(a) and (b) converges to the white (black) region in Figs. 10(c) and (d). The nonlinear MEMS resonator can be used as the memory device by storing the output of OR gate. The single MEMS resonator combines the function of OR gate and memory at $V_{\mathrm{dcn}}=210 \mathrm{mV}$.

\subsection{AND gate and memory operations}

This section numerically discusses to reprogram logic function of the logicmemory device of the single MEMS resonator. The logic function is programmed dynamically by adjusting the resonator's operating parameters. In previous section, the dc bias voltage $V_{\mathrm{dcn}}$ was set at $210 \mathrm{mV}$. Here, the dc bias voltage is reset at $120 \mathrm{mV}$.

By using the same method as shown in the previous section, we consider the nonlinear dynamics with control input. Figs. 11(a) and (b) show the amplitude

Table 2: Truth table of OR gate.

\begin{tabular}{cc|c} 
Input & & Output \\
$L_{\text {inn1 }}$ & $L_{\text {inn2 }}$ & $x$ \\
\hline 0 & 0 & "0" \\
0 & 1 & "1" \\
1 & 0 & "1" \\
1 & 1 & "1" \\
\hline
\end{tabular}




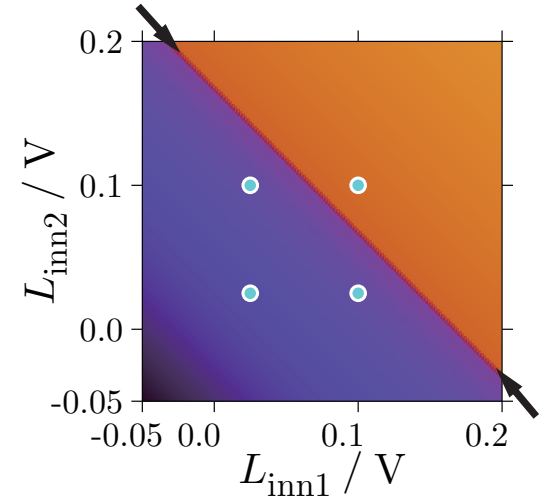

(a)

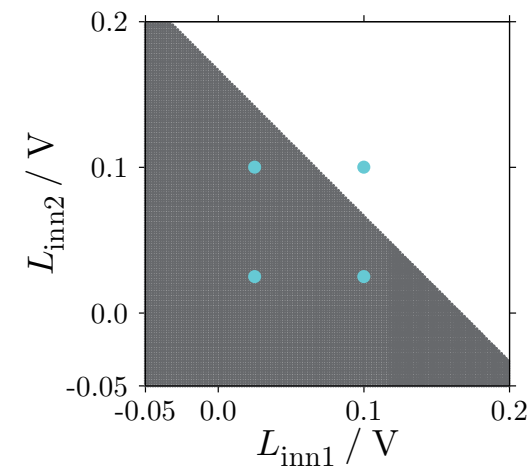

(c)

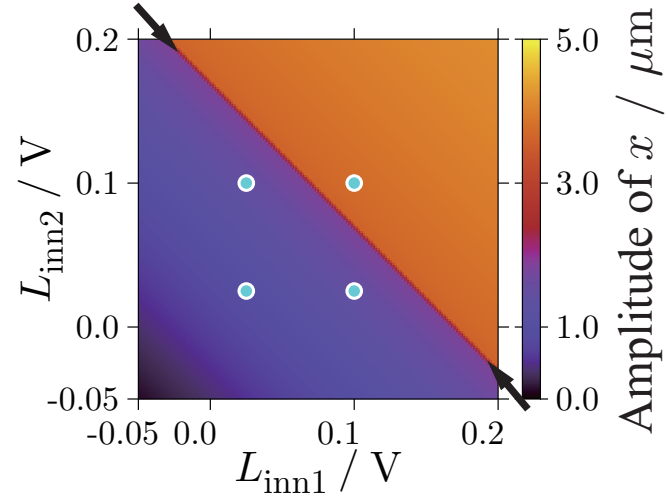

(b)

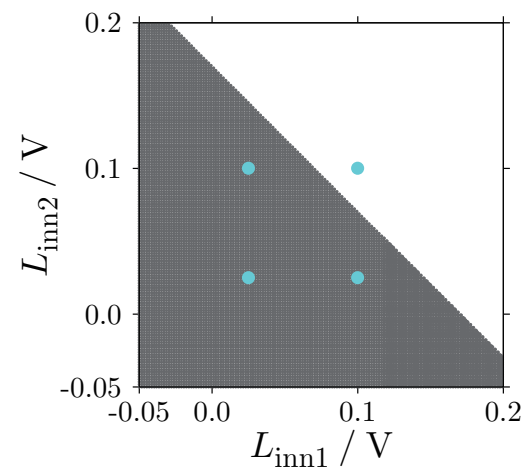

(d)

Figure 11: Logic output with and without control input at $V_{\mathrm{dcn}}=120 \mathrm{mV}$. (a) and (b) ((c) and $(d)$ ) show the amplitude modulation in the presence of the control input (the final state without the control input), as in Fig. 10: (a) Initial state is set to the logical "1". (b) Initial state corresponds to the logical " 0 ". (c) Convergence conditions corresponding to Fig. 11(a). (d) Convergence conditions corresponding to Fig. 11(b).

modulation varied in input signals. In these figures, the blue region corresponding to logical "0" increases. As in Fig. 10, the right (aqua) circles show the logic input. When the logic inputs are $(0,0),(0,1)$, or $(1,0)$, the output of the device becomes a logical " 0 " at $V_{\mathrm{dcn}}=120 \mathrm{mV}$. When the logic inputs are set to $(1,1)$, the output is a logical " 1 " under these conditions. Therefore, by changing the excitation amplitude, the nonlinear MEMS resonator works as an AND gate as shown in Tab. 3. 
Table 3: Truth table of AND gate.

\begin{tabular}{cc|c} 
Input & & Output \\
$L_{\text {inn1 }}$ & $L_{\text {inn2 }}$ & $x$ \\
\hline 0 & 0 & "0" \\
0 & 1 & "0" \\
1 & 0 & "0" \\
1 & 1 & "1" \\
\hline
\end{tabular}

Similar to the previous section, we discuss the nonlinear dynamics without the control input. As shown in Figs. 11(c) and (d), the MEMS resonator in the absence of the control input maintains its original logic output. The light region converges to the white region and the blue region corresponds to the black region. The MEMS resonator can be used as memory. The transition is slow in the line region indicated by the arrows compared to other region in Figs. 10 and 11. When the operating point of resonator is perturbed by noise [52], it is anticipated that desired logic and memory operations may not be achieved. Nevertheless, we numerically demonstrate the reprogrammable logic function that consists of OR/AND gate and the memory functions in the single nonlinear MEMS resonator due to the adjustment of the excitation amplitude.

\section{Reprogramming logic-memory operation}

Based on both experiments and numerical simulations, this section focuses on a reprogrammable logic-memory operation demonstrated in a single MEMS resonator. These numerical and experimental operations are confirmed for the behavior of device at clock evolution. In digital computation, the clock signal is commonly used and has high and low states [5, 53].

The calculated time evolutions of the device are shown in Fig. 12 and the corresponding experimental results are shown in Fig. 13. Here, the dc bias voltage $V_{\mathrm{dcn}}\left(V_{\mathrm{dce}}\right)$ is set at either $210 \mathrm{mV}$ or $120 \mathrm{mV}(110 \mathrm{mV}$ or $50 \mathrm{mV})$ 
in our numerical simulations (experiments). Figs. 12(a) and (b) (Figs. 13(a) and (b)) show the calculated time evolutions of the device at $V_{\mathrm{dcn}}=210 \mathrm{mV}$ and $V_{\mathrm{dcn}}=120 \mathrm{mV}\left(V_{\mathrm{dce}}=110 \mathrm{mV}\right.$ and $\left.V_{\mathrm{dce}}=50 \mathrm{mV}\right)$, respectively. In these figures, the purple, green, yellow, aqua and black lines show the output of the device, control input, the first logic input, the second logic input, and clock signal, respectively. When the clock signal is set at low (high) level, the control input is not (is) applied to the MEMS resonator. Note that the nonlinear MEMS resonator works as a memory (logic) device at low (high) clock signal. In Figs. 12 and 13, the logic inputs of numerical input signals $\left(L_{\mathrm{inn} 1}, L_{\mathrm{inn} 2}\right)$ and experimental input signals $\left(L_{\text {ine1 }}, L_{\text {ine2 }}\right)$ start from $(0,0)$ and continue to $(0$, $1),(1,0)$, and $(1,1)$.

As shown in Fig. 12(a) (12(b)), the nonlinear MEMS resonator works as an OR (AND) gate at $V_{\mathrm{dcn}}=210 \mathrm{mV}\left(V_{\mathrm{dcn}}=120 \mathrm{mV}\right)$ when the control input becomes high clock signal. In addition, when the control input is off at low clock signal, the nonlinear MEMS resonator can be used as the memory device by storing the output of OR (AND) gate at $V_{\mathrm{dcn}}=210 \mathrm{mV}\left(V_{\mathrm{dcn}}=120 \mathrm{mV}\right)$. The calculated results in Fig. 12 are consistent with the experimental time evolution in Fig. 13. As shown in Fig. 13(a) (13(b)), the nonlinear MEMS resonator experimentally works as an OR (AND) gate and then store the output of gate at $V_{\text {dce }}=110 \mathrm{mV}\left(V_{\text {dce }}=50 \mathrm{mV}\right)$. By adjusting the excitation amplitude, we numerically and experimentally realize the reprogrammable OR/AND gate and the memory functions in the single nonlinear MEMS resonator.

\section{Conclusion}

This study addressed a nonlinear MEMS resonator and its application to memory and reprogrammable logic functions. A schematic of a fabricated combdrive MEMS resonator was shown. It was experimentally confirmed that the MEMS resonator can be equipped with a comb drive that normally serves as a forcing actuator, but which simultaneously serves as a displacement sensor in the differential measurement. The fabricated MEMS resonator had nonlinear 


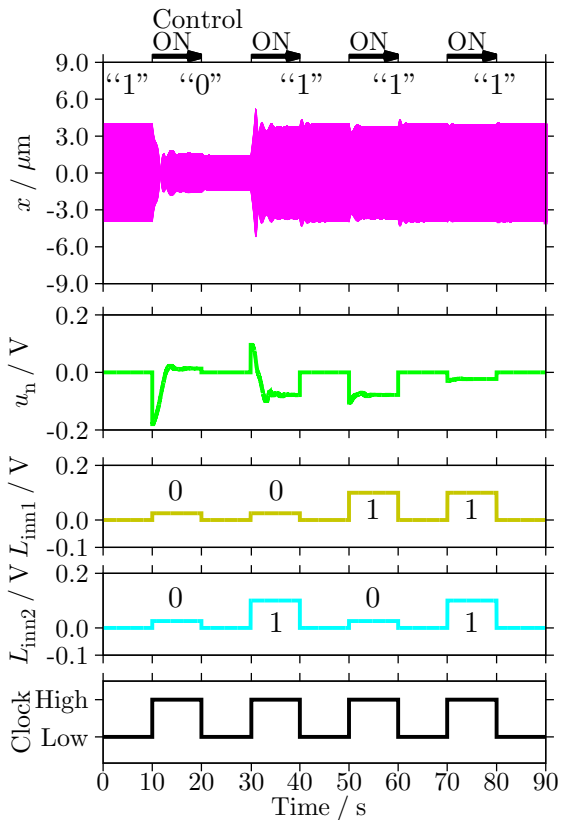

(a)

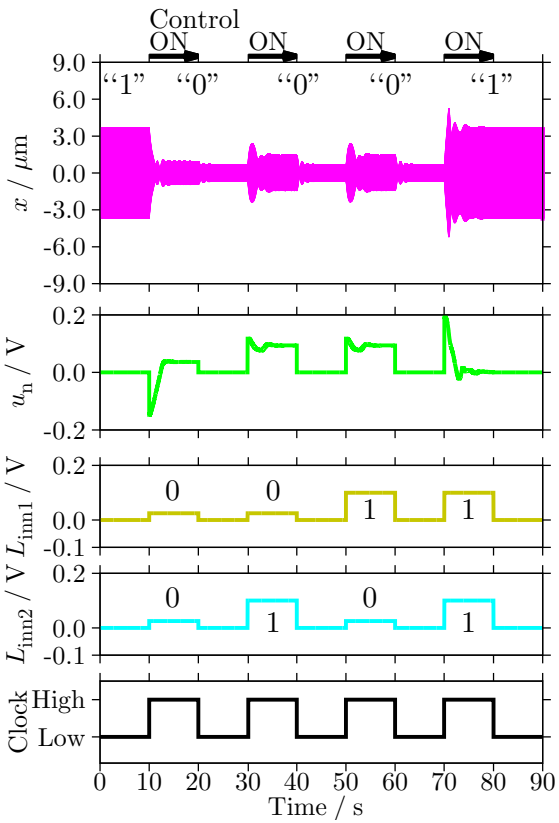

(b)

Figure 12: Time evolution of the reprogrammable logic-memory device (numerical results). When the logic inputs are $(0,0)$, the output of the device becomes a logical "0" at $V_{\mathrm{dcn}}=$ $210 \mathrm{mV}$. When the logic inputs are set at $(0,1),(1,0)$, or $(1,1)$, the output is a logical "1" at $V_{\mathrm{dcn}}=210 \mathrm{mV}$. On the other hands, at $V_{\mathrm{dcn}}=120 \mathrm{mV}$, when the logic inputs are set at $(0$, $1)$ or $(1,0)$, the output is a logical " 0 ": (a) OR gate and memory functions $\left(V_{\mathrm{dcn}}=210 \mathrm{mV}\right)$. (b) AND gate and memory functions $\left(V_{\mathrm{dcn}}=120 \mathrm{mV}\right)$.

responses. The dynamical model of such a resonator was described by the Duffing equation. The numerical and experimental results suggested that the fabricated nonlinear MEMS resonator can be used as memory and logic devices with a binary representation.

A logic-memory device, which consists of memory and multiple-input gate, was demonstrated in the nonlinear MEMS resonator. The nonlinear dynamics in the presence (absence) of control input was examined as the logic (memory) operation. When the control input was applied to the MEMS resonator with feedback system, two equal-amplitude regions existed. When the control input was off, the nonlinear MEMS resonator maintained its original logical state. 


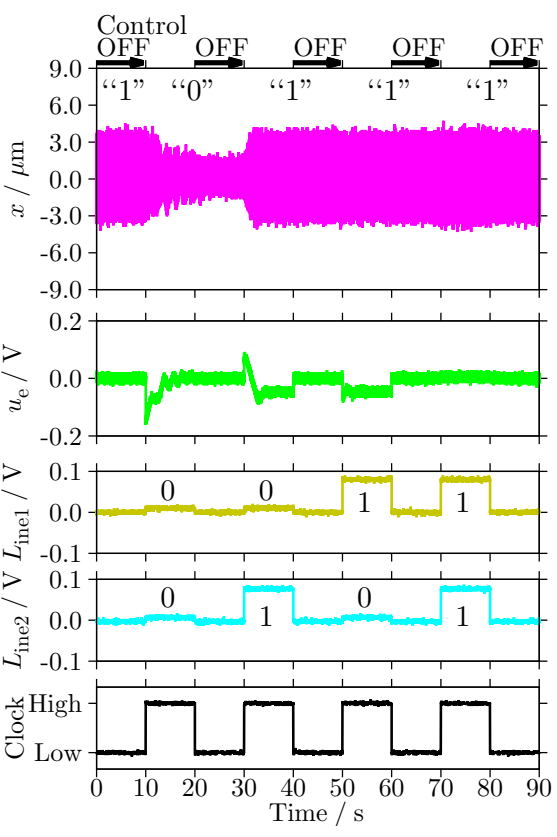

(a)

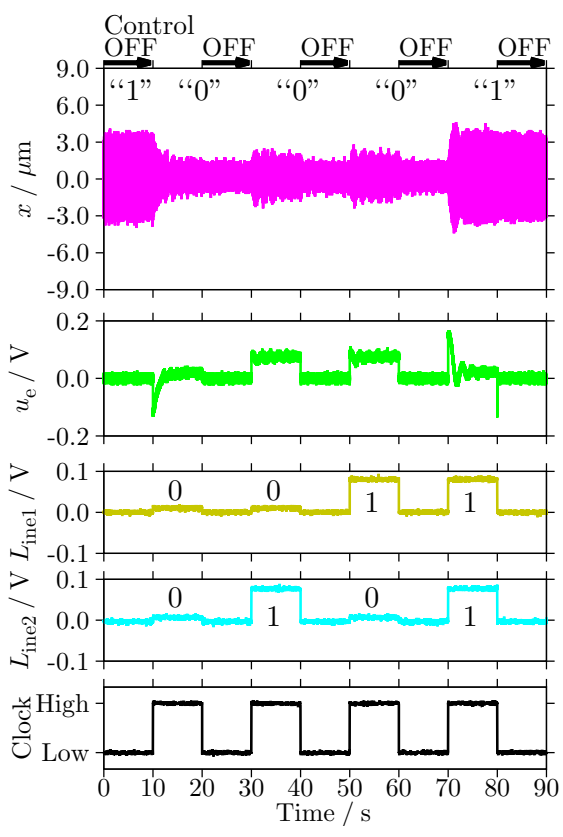

(b)

Figure 13: Corresponding experimental time evolution of the reprogrammable logic-memory device. In our experiments, the logic inputs $(0,0)$ of experimental input signals ( $\left.L_{\text {ine1 }}, L_{\text {ine2 }}\right)$ have a voltage of $20 \mathrm{mV},(0,1)$ and $(1,0)$ have a voltage of $90 \mathrm{mV}$, and finally $(1,1)$ have $160.0 \mathrm{mV}$. In our experiments, based on our numerical simulations, the excitation amplitude, gain, and logic inputs are swept within the operating range and are adjusted: (a) OR gate and memory functions at $V_{\text {dce }}=110 \mathrm{mV}$. (b) AND gate and memory functions at $V_{\text {dce }}=50$ $\mathrm{mV}$.

A device, which combines AND or OR gate and memory functions operating at room temperature, was numerically demonstrated in the single MEMS resonator.

Through the experiments and numerical simulations, a multifunctional device of the nonlinear MEMS resonator was investigated. This study numerically and experimentally realized the reprogrammable logic function that consists of OR/AND gate by adjusting the excitation amplitude and the memory function by storing logic information in the single nonlinear MEMS resonator.

Important features of the logic gate are a switching operation speed and 
an integration density. CMOS (Complementary Metal-Oxide-Semiconductor) devices that consist of PMOS and NMOS transistors are growing in popularity because of their integrated system and high speed (more than $1 \mathrm{GHz}$ ) [5, 53]. The logic-memory device consisted of the resonator and electric circuits that relate to feedback control and measurement system. Therefore, in our logic and memory system based on the resonator, the operating speed depended not only on $Q$ factor but also on the time constant of the electric circuits and then the duration of the switching was about $5 \mathrm{~s}$. The analog multiplier and the operational amplifier was used as electric circuits to perform the control and measurement. The device integration will be performed by using an on-chip device in the future.

Nevertheless, we fabricated the multifunction device that can offer the reprogrammable logic-memory operation. Therefore, by using one voltage source per bit, it is possible to include additional functions such as $n$-bit OR/AND gate [38] and memory devices in the single resonator. Furthermore, the demonstration of this reprogrammable logic-memory device opens the way to further research in the realization of an on-demand device [54] with multi-functions based on the nonlinear MEMS resonator. Based on our logic-memory results, it may be possible to realize mechanical neurocomputing [55] in the coupled MEMS resonators.

\section{Acknowledgement}

We are grateful to Prof. S. Naik (Weber State University, USA) for his support in the design of MEMS resonators. This work was partly supported by the Global COE of Kyoto University, Regional Innovation Cluster Program "Kyoto Environmental Nanotechnology Cluster", the JSPS KAKENHI (Grantin-Aid for Exploratory Research) $\sharp 21656074$, and the Grant-in-Aid for JSPS Fellows $\sharp 26462$. 


\section{References}

[1] H. H. Goldstine, The computer from Pascal to von Neumann, Princeton University Press, 1972.

[2] P. E. Ceruzzi, Computing: A Concise History, MIT Press, 2012.

[3] J. Magnuson, P. C. Fu, Public Health Informatics and Information Systems, Springer, 2014.

[4] C. Eames, R. Eames, A Computer Perspective: Background to the Computer Age, Harvard University Press, 1990.

[5] D. M. Harris, S. L. Harris, Digital design and computer architecture, Morgan Kaufmann, 2007.

[6] B. Collier, J. MacLachlan, Charles Babbage: And the Engines of Perfection, Oxford University Press, 2000.

[7] G. O'Regan, A brief history of computing 2nd ed., Springer, 2012.

[8] L. M. Roylance, J. B. Angell, A batch-fabricated silicon accelerometer, IEEE Transactions on Electron Devices 26 (12) (1979) 1911-1917.

[9] E. Bassous, H. H. Taub, L. Kuhn, Ink jet printing nozzle arrays etched in silicon, Applied Physics Letters 31 (2) (1977) 135-137.

[10] U. Shah, M. Sterner, J. Oberhammer, High-directivity mems-tunable directional couplers for 10-18-ghz broadband applications, IEEE Transactions on Microwave Theory and Techniques 61 (9) (2013) 3236-3246.

[11] N. Somjit, G. Stemme, J. Oberhammer, Power handling analysis of highpower-band all-silicon mems phase shifters, IEEE Transactions on Electron Devices 58 (5) (2011) 1548-1555.

[12] M. Sterner, N. Roxhed, G. Stemme, J. Oberhammer, Static zero-powerconsumption coplanar waveguide embedded dc-to-rf metal-contact mems 
switches in two-port and three-port configuration, IEEE Transactions on Electron Devices 57 (7) (2010) 1659-1669.

[13] J. A. Pelesko, D. H. Bernstein, Modeling MEMS and NEMS, CHAMPMAN and HALL/CRC, 2003.

[14] V. Kaajakari, Practical MEMS, Small Gear Publishing, 2009.

[15] H. C. Nathanson, R. A. Wickstrom, A resonant-gate silicon surface transistor with high-q band-pass properties, Applied Physics Letters 7 (4) (1965) $84-86$.

[16] H. C. Nathanson, W. E. Newell, R. A. Wickstrom, J. R. Davis Jr, The resonant gate transistor, IEEE Transactions on Electron Devices 14 (3) (1967) 117-133.

[17] R. L. Badzey, G. Zolfagharkhani, A. Gaidarzhy, P. Mohanty, A controllable nanomechanical memory element, Applied Physics Letters 85 (16) (2004) $3587-3589$.

[18] R. L. Badzey, P. Mohanty, Coherent signal amplification in bistable nanomechanical oscillators by stochastic resonance, Nature (London) 437 (7061) (2005) 995-998.

[19] S. C. Masmanidis, R. B. Karabalin, I. De Vlaminck, G. Borghs, M. R. Freeman, M. L. Roukes, Multifunctional nanomechanical systems via tunably coupled piezoelectric actuation, Science 317 (5839) (2007) 780-783.

[20] I. Mahboob, H. Yamaguchi, Bit storage and bit flip operations in an electromechanical oscillator, Nature Nanotechnology 3 (5) (2008) 275-279.

[21] D. N. Guerra, A. R. Bulsara, W. L. Ditto, S. Sinha, K. Murali, P. Mohanty, A noise-assisted reprogrammable nanomechanical logic gate, Nano Letters 10 (4) (2010) 1168-1171. 
[22] H. Noh, S.-B. Shim, M. Jung, Z. G. Khim, J. Kim, A mechanical memory with a dc modulation of nonlinear resonance, Applied Physics Letters 97 (3) (2010) 033116-1-033116-3.

[23] Q. P. Unterreithmeier, T. Faust, J. P. Kotthaus, Nonlinear switching dynamics in a nanomechanical resonator, Physical Review B 81 (24) (2010) 241405-1-241405-4.

[24] I. Mahboob, E. Flurin, K. Nishiguchi, A. Fujiwara, H. Yamaguchi, Interconnect-free parallel logic circuits in a single mechanical resonator, Nature Communications 2 (2011) 198.

[25] A. Yao, T. Hikihara, Switching control between stable periodic vibrations in a nonlinear mems resonator, Proc. of NOLTA2011 (2011) 709-712.

[26] D. Hatanaka, I. Mahboob, H. Okamoto, K. Onomitsu, H. Yamaguchi, An electromechanical membrane resonator, Applied Physics Letters 101 (6) (2012) 063102-1-063102-5.

[27] A. Yao, T. Hikihara, Reading and writing operations of memory device in micro-electromechanical resonator, IEICE Electronics Express 9 (14) (2012) 1230-1236.

[28] A. Yao, T. Hikihara, Read and write operations of memory device consisting of nonlinear mems resonator, Proc. of NOLTA2012 (2012) 352-355.

[29] N. A. Khovanova, J. Windelen, Minimal energy control of a nanoelectromechanical memory element, Applied Physics Letters 101 (2) (2012) 024104$1-024104-4$.

[30] J.-S. Wenzler, T. Dunn, T. Toffoli, P. Mohanty, A nanomechanical fredkin gate, Nano letters 14 (1) (2013) 89-93.

[31] A. Uranga, J. Verd, E. Marigó, J. Giner, J. L. Muñóz-Gamarra, N. Barniol, Exploitation of non-linearities in cmos-nems electrostatic resonators for mechanical memories, Sensors and Actuators A: Physical 197 (2013) 88-95. 
[32] A. Yao, T. Hikihara, Counter operation in nonlinear micro-electromechanical resonators, Physics Letters A 377 (38) (2013) 2551-2555.

[33] A. Yao, T. Hikihara, Logical behavior in memory devices of coupled nonlinear mems resonators, Proc. of NOLTA2013 (2013) 30-33.

[34] I. Mahboob, M. Mounaix, K. Nishiguchi, A. Fujiwara, H. Yamaguchi, A multimode electromechanical parametric resonator array, Scientific reports 4 (4448) (2014) 1-8.

[35] A. Yao, T. Hikihara, Logic-memory device of a mechanical resonator, Applied Physics Letters 105 (12) (2014) 123104-1-123104-4.

[36] A. Yao, T. Hikihara, Reprogrammable logic-memory operation in a nonlinear mems resonator, Proc. of NOLTA2015 (2015) 736-739.

[37] A. Yao, Logic and memory devices of nonlinear microelectromechanical resonator, Ph.D. thesis, Kyoto University (2015).

[38] M. A. A. Hafiz, L. Kosuru, M. I. Younis, Microelectromechanical reprogrammable logic device, Nature Communications 7 (2016) 11137.

[39] M. V. Andres, K. W. H. Foulds, M. J. Tudor, Nonlinear vibrations and hysteresis of micromachined silicon resonators designed as frequency-out sensors, Electronics Letters 23 (18) (1987) 952-954.

[40] A. Frangi, Advances in multiphysics simulation and experimental testing of MEMS, Vol. 2, World Scientific Publishing, 2008.

[41] J. F. Rhoads, S. W. Shaw, K. L. Turner, Nonlinear dynamics and its applications in micro-and nanoresonators, Journal of Dynamic Systems, Measurement, and Control 132 (3) (2010) 034001.

[42] R. M. C. Mestrom, R. H. B. Fey, J. T. M. van Beek, K. L. Phan, H. Nijmeijer, Modelling the dynamics of a MEMS resonator: Simulations and experiments, Sensors and Actuators A: Physical 142 (1) (2008) 306-315. 
[43] S. Naik, T. Hikihara, H. Vu, A. Palacios, V. In, P. Longhini, Local bifurcations of synchronization in self-excited and forced unidirectionally coupled micromechanical resonators, Journal of Sound and Vibration 331 (5) (2012) $1127-1142$.

[44] D. Antonio, D. H. Zanette, D. López, Frequency stabilization in nonlinear micromechanical oscillators, Nature Communications 3 (2012) 806.

[45] S. Naik, T. Hikihara, Characterization of a MEMS resonator with extended hysteresis, IEICE Electronics Express 8 (5) (2011) 291-298.

[46] S. Naik, Investigation of synchronization in a ring of coupled MEMS resonators, Ph.D. thesis, Kyoto University (2011).

[47] A. Cowen, G. Hames, D. Monk, S. Wilcenski, B. Hardy, Soimumps design handbook, MEMSCAP Inc.

[48] T. S. Parker, L. Chua, Practical numerical algorithms for chaotic systems, Springer Science \& Business Media, 2012.

[49] H. B. Stewart, J. M. Thompson, Nonlinear Dynamics and Chaos, John Wiley and Sons, 1986.

[50] J. Guckenheimer, P. Holmes, Nonlinear Oscillations, Dynamical Systems, and Bifurcations of Vector Fields, Springer, 1983.

[51] Y. Ueda, Theory of Chaotic Phenomena, Corona publishing co., ltd., 2008 (in Japanese).

[52] J. Zou, S. Buvaev, M. Dykman, H. B. Chan, Poisson noise induced switching in driven micromechanical resonators, Physical Review B 86 (15) (2012) 155420 .

[53] R. L. Tokheim, Schaum's outline of theory and problems of digital principles, McGraw-Hill, 1994. 
[54] R. Yang, K. Terabe, G. Liu, T. Tsuruoka, T. Hasegawa, J. K. Gimzewski, M. Aono, On-demand nanodevice with electrical and neuromorphic multifunction realized by local ion migration, ACS nano 6 (11) (2012) 9515-9521.

[55] F. C. Hoppensteadt, E. M. Izhikevich, Synchronization of mems resonators and mechanical neurocomputing, IEEE Transactions on Circuits and Systems I: Fundamental Theory and Applications 48 (2) (2001) 133-138. 